\title{
Engineering the Yeast Saccharomyces cerevisiae for the Production of L-(+)-Ergothioneine
}

\begin{abstract}
Steven A. van der Hoek ${ }^{1}$, Behrooz Darbani ${ }^{1}$, Karolina E. Zugaj ${ }^{1}$, Bala Krishna Prabhala ${ }^{1 \dagger}$, Mathias Bernfried Biron ${ }^{1}$, Milica Randelovic ${ }^{1}$, Jacqueline B. Medina ${ }^{1}$, Douglas B. Kell ${ }^{1,2 *}$ and Irina Borodina ${ }^{1 *}$

${ }^{1}$ The Novo Nordisk Foundation Center for Biosustainability, Technical University of Denmark, Kongens Lyngby, Denmark, ${ }^{2}$ Department of Biochemistry, Institute of Integrative Biology, University of Liverpool, Liverpool, United Kingdom
\end{abstract}

\section{OPEN ACCESS}

Edited by:

Nils Jonathan Helmuth Averesch, Stanford University, United States

Reviewed by:

Tsutomu Tanaka

Kobe University, Japan Jingwen Zhou,

Jiangnan University, China

${ }^{*}$ Correspondence: Douglas B. Kell

douke/@biosustain.dtu.dk Irina Borodina

irbo@biosustain.dtu.dk

tPresent address: Bala Krishna Prabhala, Faculty of Science, Institute of Physics, Chemistry and Pharmacy, University of Southern Denmark,

Odense, Denmark

Specialty section:

This article was submitted to

Bioprocess Engineering,

a section of the journal

Frontiers in Bioengineering and

Biotechnology

Received: 21 May 2019

Accepted: 26 September 2019

Published: 11 October 2019

Citation:

van der Hoek SA, Darbani $B$ Zugaj KE, Prabhala BK, Biron MB, Randelovic M, Medina JB, Kell DB and Borodina I (2019) Engineering the

Yeast Saccharomyces cerevisiae for the Production of L-(+)-Ergothioneine.

Front. Bioeng. Biotechnol. 7:262.

doi: 10.3389/fbioe.2019.00262
L-(+)-Ergothioneine (ERG) is an unusual, naturally occurring antioxidant nutraceutical that has been shown to help reduce cellular oxidative damage. Humans do not biosynthesise ERG, but acquire it from their diet; it exploits a specific transporter (SLC22A4) for its uptake. ERG is considered to be a nutraceutical and possible vitamin that is involved in the maintenance of health, and seems to be at too low a concentration in several diseases in vivo. Ergothioneine is thus a potentially useful dietary supplement. Present methods of commercial production rely on extraction from natural sources or on chemical synthesis. Here we describe the engineering of the baker's yeast Saccharomyces cerevisiae to produce ergothioneine by fermentation in defined media. After integrating combinations of ERG biosynthetic pathways from different organisms, we screened yeast strains for their production of ERG. The highest-producing strain was also engineered with known ergothioneine transporters. The effect of amino acid supplementation of the medium was investigated and the nitrogen metabolism of $S$. cerevisiae was altered by knock-out of TOR1 or YIH1. We also optimized the media composition using fractional factorial methods. Our optimal strategy led to a titer of $598 \pm 18 \mathrm{mg} / \mathrm{L}$ ergothioneine in fed-batch culture in $1 \mathrm{~L}$ bioreactors. Because $S$. cerevisiae is a GRAS ("generally recognized as safe") organism that is widely used for nutraceutical production, this work provides a promising process for the biosynthetic production of ERG.

Keywords: ergothioneine, metabolic engineering, medium optimization, Saccharomyces cerevisiae, yeast, nutraceutical

\section{INTRODUCTION}

Ergothioneine (ERG) (2-mercaptohistidine trimethylbetaine, IUPAC name (2S)-3-(2-Thioxo2,3-dihydro-1H-imidazol-4-yl)-2-(trimethylammonio)propanoate) is a naturally occurring antioxidant that can be found universally in plants and mammals (Melville, 1959); it possesses a tautomeric structure, but is mainly present in the thione form at physiological $\mathrm{pH}$ (Figure 1). Ergothioneine was discovered in 1909 in the ergot fungus Claviceps purpurea (Tanret, 1909), and its structure was determined 2 years later (Barger and Ewins, 1911). Subsequently, several other organisms were found to produce ergothioneine, including the filamentous fungus Neurospora crassa (Genghof et al., 1956), the yeast Schizosaccharomyces pombe (Pluskal et al., 2014), various actinobacteria (Genghof, 1970) including Mycobacterum smegmatis (Seebeck, 2010), 


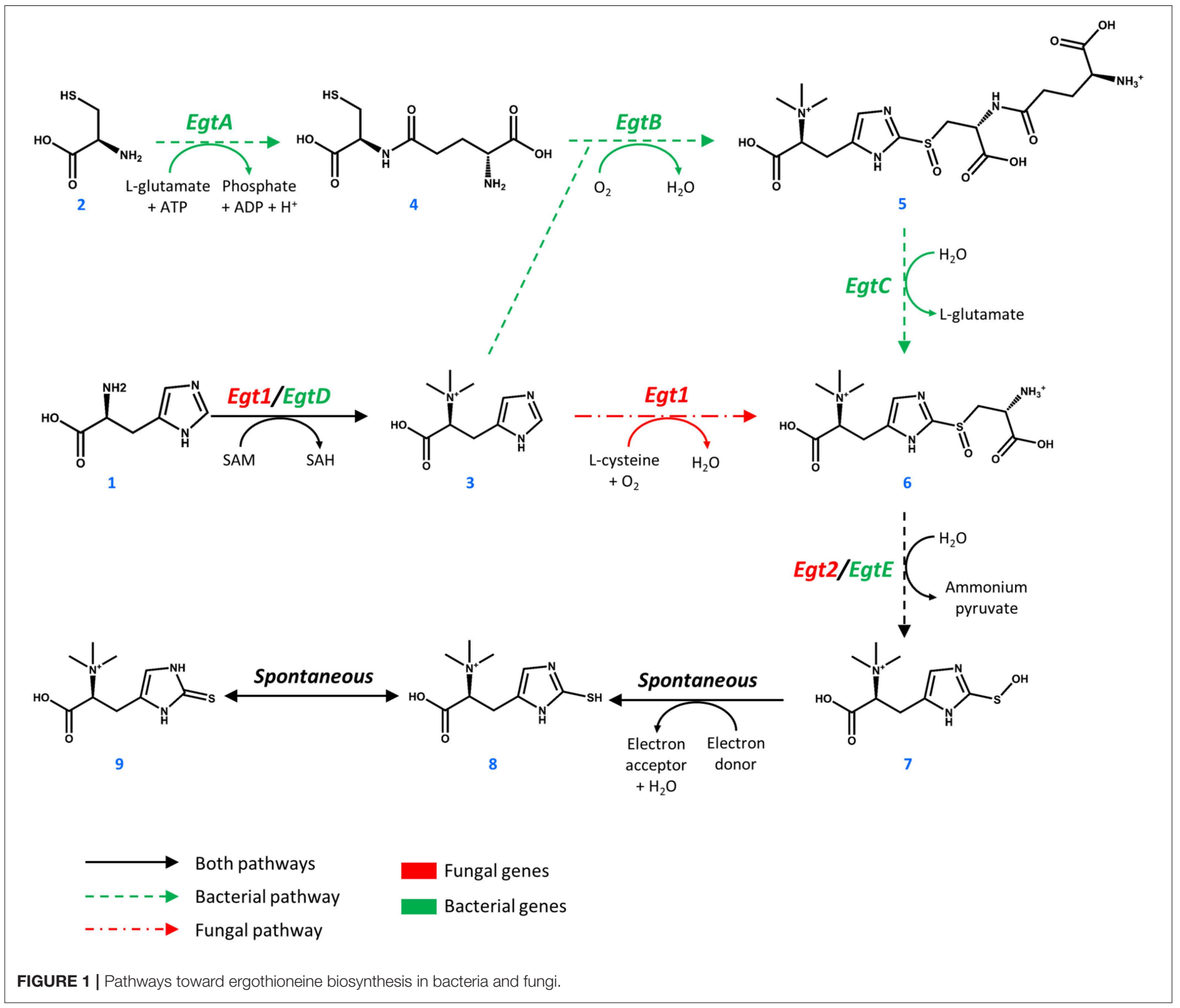

and in particular various basidiomycetes (mushrooms) (Genghof, 1970). Higher eukaryotes have been shown to contain ergothioneine (Melville, 1959; Halliwell et al., 2018), but to date no higher eukaryotes have been reported to biosynthesize ergothioneine. Plants are thought to take ergothioneine excreted by fungi up from the soil (Audley and Tan, 1968; Tan and Audley, 1968) or through symbiotic relationships (Park et al., 2010; Guo et al., 2016).

Ergothioneine is synthesized from one molecule of L-histidine (1), one molecule of L-cysteine (2), and 3 methyl groups donated by S-adenosyl-L-methionine (SAM, Figure 1). In M. smegmatis, the reaction sequence is catalyzed by five enzymes, encoded by egtA-E genes arranged in an operon (Sao Emani et al., 2018).

Abbreviations: ERG, L-(+)-ergothioneine; HCO, S-(hercyn-2-yl)-L-cysteine Soxide; PBS, phosphate-buffered saline; PI, propidium iodide; PLP, pyridoxal $5^{\prime}$ phosphate; SAM, S-adenosyl-L-methionine.
Four enzymes of the cluster EgtA, EgtB, EgtC, and EgtD catalyze four individual reactions that methylate L-histidine to form hercynine (3), convert L-cysteine to $\gamma$-L-glutamyl-L-cysteine (4), combine hercynine and $\gamma$-L-glutamyl-L-cysteine to generate $\gamma$ L-glutamyl-S-(hercyn-2-yl)-L-cysteine S-oxide (5) and produce the S-(hercyn-2-yl)-L-cysteine S-oxide (6, HCO). In fungi, the biosynthetic pathway is different, as a single enzyme Egt1 catalyzes the methylation of L-histidine (1) to give hercynine (3), which in turn is sulfoxidized with cysteine, producing HCO (6). HCO is converted into 2-(hydroxysulfanyl)hercynine (7) by a $\beta$-lyase, encoded by egtE in $M$. smegmatis and by the EGT2 gene in fungi. This compound is spontaneously reduced to ergothioneine (8, thiol form and $\mathbf{9}$, thione form). Comparing the bacterial and fungal pathway, it is important to note that the utilization of glutamate in the ergothioneine biosynthesis pathway also requires ATP. Recently, enzymes for the anaerobic production of ergothioneine were found in Chlorobium limicola 
(Burn et al., 2017), where sulfur is directly transferred to the imidazole ring of hercynine to form ergothioneine (Leisinger et al., 2019). However, it is suggested that the anaerobic C-S bond formation is less efficient than it's aerobic counterpart (Burn et al., 2017).

The antioxidant properties of ERG include the scavenging of free radicals and reactive oxygen species (Akanmu et al., 1991; Park et al., 2010; Ta et al., 2011) and the chelation of divalent metal ions (Hanlon, 1971). ERG has been shown to reduce oxidative damage in a variety of mammals (Deiana et al., 2004; Cheah and Halliwell, 2012). In humans, ERG is mainly accumulated in the liver, the kidneys, in erythrocytes, the eye lens, and in seminal fluid (Leone and Mann, 1951; Melville et al., 1954; Shires et al., 1997). It is transported by a specific transporter SLC22A4 (previously known as OCTN1) (Grundemann et al., 2005; Tschirka et al., 2018). The natural selection of such a transporter implies that ergothioneine is involved in the maintenance of health or the mitigation of disease, and it may even be a vitamin (Paul and Snyder, 2010). ERG has demonstrated effects in in vivo models of several neurodegenerative diseases (Link, 1995; Yang et al., 2012; Song et al., 2014), in ischemia reperfusion injury (Bedirli et al., 2004; Sakrak et al., 2008a,b), and in a variety of other diseases (Halliwell et al., 2018). Ergothioneine accumulates at sites of injury through the upregulation of SLC22A4/OCTN1 (Cheah et al., 2016; Tang et al., 2016). It is only slowly metabolized and excreted in humans (Cheah et al., 2017).

Because humans cannot produce ERG, they must obtain it through their diet. Although plants and animals also accumulate it to some degree, the main natural dietary source of ERG is basidiomycete mushrooms, where some species contain up to $7 \mathrm{mg}$ of ERG per gram dry weight (Ey et al., 2007; Pfeiffer et al., 2011; Kalaras et al., 2017; Halliwell et al., 2018). Because of the beneficial effects and possible involvement of ERG in disease, ergothioneine may potentially prove its value in the global dietary supplement market, which was estimated at some $\$ 241.1$ billion in 2019 (Wang et al., 2016). Currently, commercial ergothioneine is extracted from mushrooms or synthesized chemically.

Production of ergothioneine in microbial cell factories would provide a sustainable low-cost alternative to its current manufacturing processes. So far, fermentative ERG production has been reported in bacteria and filamentous fungi, including Methylobacterium aquaticum strain 22A (Alamgir et al., 2015), Aureobasidium pullulans (Fujitani et al., 2018), Rhodotorula mucilaginosa (Fujitani et al., 2018), cyanobacteria (Pfeiffer et al., 2011), Aspergillus oryzae (Takusagawa et al., 2019), and Escherichia coli (Osawa et al., 2018; Tanaka et al., 2019). Engineering of $M$. aquaticum with an extra copy of egtBD and the deletion of hutH (histidine-ammonia-lyase gene) lead to a strain that produced $7.0 \mathrm{mg}$ ERG/g dry cell weight and 100 $\mu \mathrm{g}$ ERG/5 mL/7 days (Fujitani et al., 2018). In another study, multiple copies of EGT1 and EGT2 genes from N. crassa were integrated into the genome of the filamentous fungi A. oryzae. The engineered strain produced $231 \mathrm{mg}$ ergothioneine per $\mathrm{kg}$ solid media (Takusagawa et al., 2019). In E. coli, at first $24 \mathrm{mg} / \mathrm{L}$ of secreted ERG was produced by expression of egtBCDE genes from M. smegmatis and optimization of the medium composition
(Osawa et al., 2018). This was followed up by a study, in which the authors also expressed egt $A$ and improved the strain by enhancing the cysteine and S-adenosyl methionine biosynthesis, as well as optimizing their medium further, to produce 1.3 $\mathrm{g} / \mathrm{L}$ of ergothioneine in $216 \mathrm{~h}$ by fermentation (Tanaka et al., 2019). To the best of our knowledge, there are no reports on ergothioneine production in baker's yeast, which is the preferred host for the production of nutraceuticals (Huang et al., 2008; Li and Borodina, 2014; Yuan and Alper, 2019). In this study we describe the metabolic engineering of the yeast Saccharomyces cerevisiae for the production of ergothioneine, reaching a titer of $0.6 \mathrm{~g} / \mathrm{L}$ in fed-batch fermentation for $84 \mathrm{~h}$.

\section{MATERIALS AND METHODS}

\section{Strains and Chemicals}

S. cerevisiae strain CEN.PK113-7D (MATa URA3 HIS3 LEU2 TRP1 MAL2-8 ${ }^{\text {c }}$ SUC2) was a gift from Peter Kötter (Goethe University, Frankfurt/Main, Germany). S. pombe strain DSM 70572, obtained from Leibniz-Institut DSMZ-Deutsche Sammlung von Mikroorganismen und Zeilkulturen $\mathrm{GmbH}$ (Germany) was used for genomic DNA extraction. E. coli DH5 $\alpha$ was used for cloning. Ergothioneine (catalog \#E7521, $\geq 98 \%$ purity) was from Sigma-Aldrich, hercynine (catalog \# H288900, $\geq 95 \%$ purity) was from Toronto Research Chemicals Inc. Simulated fed-batch medium components (EnPump 200) were from EnPresso GmbH (Germany). These components consist of a polysaccharide, in powder form, to add to the medium and an enzyme to release glucose from the polysaccharide. The rate of release is dependent on the enzyme concentration and allows for simulated carbon-limited fermentation.

\section{Cloning}

CpEgt1, MsEgtA, and MsEgtE were codon-optimized for $S$. cerevisiae using GeneGenie (Swainston et al., 2014), while the other genes were codon-optimized for S. cerevisiae using the codon-optimization tool provided by GeneArt. The genes were then ordered as synthetic gene strings from IDT DNA (MsEgtA) or GeneArt (all other synthetic gene strings). The only exceptions were two genes from $S$. pombe, which were isolated from genomic DNA. The DNA sequences of the genes are in Supplementary Table 1. All yeast strain construction was done using CRISPR/Cas9 and EasyClone-MarkerFree methods (Stovicek et al., 2015; Jessop-Fabre et al., 2016). Correct cloning was validated by sequencing (Eurofins Genomics). Correct genome modification of yeasts was validated by colony PCR. The details on primers (Supplementary Tables 2, 3), biobricks (Supplementary Table 4), plasmids (Supplementary Tables 5, 6), and strains (Supplementary Table 7) are in Supplementary Materials.

\section{Media and Small-Scale Cultivation Conditions}

For E. coli selection, we used Lysogeny Broth (LB) with 100 $\mathrm{mg} / \mathrm{L}$ ampicillin. For the selection of yeast strains, we used YeastPeptone-Dextrose (YPD) agar supplemented with $200 \mathrm{mg} / \mathrm{L}$ G418 for selection of Cas9 vector and $100 \mathrm{mg} / \mathrm{L}$ nourseothricin 
for selection of the gRNA vector. Synthetic Complete (SC) medium was prepared using $6.7 \mathrm{~g} / \mathrm{L}$ Yeast Nitrogen Base without amino acids from Sigma-Aldrich, $1.92 \mathrm{~g} / \mathrm{L}$ Synthetic Drop-out supplement without histidine from Sigma-Aldrich and $76 \mathrm{mg} / \mathrm{L}$ histidine. For ERG production, yeast strains were cultivated in SC medium with 20 or $40 \mathrm{~g} / \mathrm{L}$ glucose or with 60 $\mathrm{g} / \mathrm{L}$ EnPump substrate (and 0.6\% enzyme reagent) as carbon source. Precultures for cultivation experiments were prepared by inoculating a single colony of a strain into $5 \mathrm{~mL}$ of the medium used in the cultivation experiment and incubating at $30^{\circ} \mathrm{C}$ and $250 \mathrm{rpm}$ for $24 \mathrm{~h}$. The cultivations were performed in 24-deepwell plates from EnzyScreen, $3 \mathrm{~mL}$ of medium was used per well and the starting $\mathrm{OD}_{600}$ was 0.5 . The plates were incubated at $30^{\circ} \mathrm{C}$ with $250 \mathrm{rpm}$ agitation.

\section{HPLC Analyses}

To determine the extracellular ergothioneine concentration, a $1 \mathrm{~mL}$ sample of fermentation broth was centrifuged at $3,000 \times$ $\mathrm{g}$ for $5 \mathrm{~min}$, the supernatant was moved into an HPLC vial and stored at $-20^{\circ} \mathrm{C}$ until the analysis. The remaining cell pellet was washed twice with $1 \mathrm{~mL}$ MilliQ water and resuspended in $1 \mathrm{~mL}$ water. The extraction of intracellular ERG was performed according to Alamgir et al. (2015) as following. The mixture was heated at $94^{\circ} \mathrm{C}$ for $10 \mathrm{~min}$, vortexed at $1,600 \mathrm{rpm}$ for 30 min using a DVX-2500 Multi-Tube Vortexer from VWR, and centrifuged at $10,000 \times \mathrm{g}$ for $5 \mathrm{~min}$. The supernatant was transferred into an HPLC vial and stored at $+4^{\circ} \mathrm{C}$ until analysis. The ERG concentrations were measured using a Dionex Ultimate 3000 HPLC system. Quantification was done based on standard curves using Chromeleon software. Five microliter of sample was injected on a Cortects UPLC T3 reversed-phase column (particle size $1.6 \mu \mathrm{m}$, pore size $120 \AA .2 .1 \times 150 \mathrm{~mm}$ ). The flow rate was $0.3 \mathrm{~mL} / \mathrm{min}$, starting with $2.5 \mathrm{~min}$ of $0.1 \%$ formic acid, going up to $70 \%$ acetonitrile, $30 \% 0.1 \%$ formic acid at $3 \mathrm{~min}$ for $0.5 \mathrm{~min}$, after which $100 \% 0.1 \%$ formic acid was run from min 4 to 9 . Ergothioneine was detected at a wavelength of $254 \mathrm{~nm}$. For analysis of bioreactor samples, we additionally quantified glucose, ethanol, pyruvate, and acetate concentrations by HPLC as described (Borodina et al., 2015).

\section{Fed-Batch Fermentation in Bioreactors}

A single colony from a YPD plate with ST8927 colonies (see below) was used to inoculate $5 \mathrm{~mL}$ of minimal media in $14 \mathrm{~mL}$ tube. The tube was incubated at $30^{\circ} \mathrm{C}$ and $250 \mathrm{rpm}$ overnight. This overnight culture was transferred into $95 \mathrm{~mL}$ mineral medium in $500 \mathrm{~mL}$ baffled shake flask. The shake flask was then incubated overnight at $30^{\circ} \mathrm{C}$ and $250 \mathrm{rpm}$. Forty milliliters of this dense culture was used to inoculate $60 \mathrm{~mL}$ mineral medium in a new $500 \mathrm{~mL}$ baffled shake flask. Two shake flasks were prepared this way. These shake flasks were incubated at $30^{\circ} \mathrm{C}$ and 250 rpm for $4 \mathrm{~h}$, the content of both shake flasks was combined, then centrifuged at $3,000 \times \mathrm{g}$ for $5 \mathrm{~min}$. The supernatant was discarded, the pellet was washed with $25 \mathrm{~mL}$ sterile water, resuspended and centrifuged as before. The supernatant was discarded and the pellet resuspended in $10 \mathrm{~mL}$ mineral medium. This was then used to inoculate $0.5 \mathrm{~L}$ mineral medium in a $1 \mathrm{~L}$ Sartorius bioreactor. The starting OD600 was 0.85 . The stirring rate was set at $500 \mathrm{rpm}$, the temperature was kept at $30^{\circ} \mathrm{C}$, and $\mathrm{pH}$ was maintained at $\mathrm{pH} 5.0$ using $2 \mathrm{M} \mathrm{KOH}$ and $2 \mathrm{M} \mathrm{H}_{2} \mathrm{SO}_{4}$. The feeding was started as soon as $\mathrm{CO}_{2}$ in the off-gas decreased by $50 \%$. The initial feed rate was set at $0.6 \mathrm{~g}$ glucose $\mathrm{h}^{-1}$, linearly increasing to $2.5 \mathrm{~g}$ glucose $\mathrm{h}^{-1}$ over the span of $25.5 \mathrm{~h}$. After that, the feed was set at a constant $1.4 \mathrm{~g}$ glucose $\mathrm{h}^{-1}$ and $17.8 \mathrm{~h}$ later, the feeding rate was set to a constant $2.9 \mathrm{~g}$ glucose $\mathrm{h}^{-1}$. The feed was stopped at $84 \mathrm{~h}$. At 60.5 and $75.5 \mathrm{~h}, 2 \mathrm{~g}\left(\mathrm{NH}_{4}\right)_{2} \mathrm{SO}_{4}$ was added as a sterile $100 \mathrm{~g} / \mathrm{L}$ solution. At 60.5 and $73.5 \mathrm{~h}, 0.5 \mathrm{~g} \mathrm{MgSO}_{4}$ was added as a sterile $50 \mathrm{~g} / \mathrm{L}$ solution, while $4 \mathrm{~mL}$ sterile trace metals solution and $2 \mathrm{~mL}$ sterile vitamin solution were added.

\section{Propidium lodide Staining and Flow Cytometry Analysis}

Precultures were prepared by inoculating a single colony of strain ST7574, ST8461, and ST8654 into separate 14-mL tubes containing $5 \mathrm{ml}$ of SC $+40 \mathrm{~g} / \mathrm{L}$ glucose $+1 \mathrm{~g} / \mathrm{L}$ His/Cys/Met and incubating at $30^{\circ} \mathrm{C}$ and $250 \mathrm{rpm}$ for $24 \mathrm{~h}$. Precultures were used to inoculate $25 \mathrm{~mL} \mathrm{SC}+40 \mathrm{~g} / \mathrm{L}$ glucose $+1 \mathrm{~g} / \mathrm{L}$ His/Cys/Met at a starting $\mathrm{OD}_{600}$ of 0.5 , which was incubated at $30^{\circ} \mathrm{C}$ and $250 \mathrm{rpm}$ for $72 \mathrm{~h}$. Every $24 \mathrm{~h}$, a $1 \mathrm{~mL}$ sample of cell culture was taken from the yeast cultivation. This sample was washed two times with $1 \mathrm{~mL}$ phosphate-buffered saline (PBS), subsequently resuspended in $0.5 \mu \mathrm{g} / \mathrm{mL}$ propidium iodide (PI) in PBS and incubated for $20 \mathrm{~min}$ at room temperature in the dark. After incubation, the cells were washed two times with PBS and the percentage of PI stained cells was determined using a MACSQuant VYB system (Miltenyi Biotec). Data analysis was performed using the FlowJo software.

\section{Fluorescent Microscopy}

Precultures were prepared by inoculating a single colony into a $14 \mathrm{~mL}$ tube containing $5 \mathrm{~mL}$ YPD medium and incubating at $30^{\circ} \mathrm{C}$ and $250 \mathrm{rpm}$ overnight. Overnight precultures of strains containing transporters linked to GFP and the control strain were used to inoculate $5 \mathrm{~mL}$ YPD medium in $14 \mathrm{~mL}$ tubes at $\mathrm{OD}_{600}=$ 2 and were cultured at $30^{\circ} \mathrm{C}$ and $250 \mathrm{rpm}$ for $5 \mathrm{~h}$. One milliliter of the culture was harvested at $3,000 \times \mathrm{g}$ for $5 \mathrm{~min}$. The cells were washed two times with PBS and subsequently pictures of the cells were taken using a Leica DM 400 B system, using Leica Application Suite V4 as image software.

\section{Medium Optimization}

A two-level fractional factorial based on the components of SC medium, with the levels high $(+1$, component 5 -folds higher than original SC medium) and low ( -1 , component 5 -folds lower than original SC medium), was used to determine the impact of individual components on the yield of ergothioneine. Two different stocks of all the individual components were prepared, one each for high and low concentrations and these were mixed together for all components to yield 64 different designed media. The design matrix for the fractional factorial grid has been attached (Supplementary Table 8). Precultures were prepared by inoculating a single colony of strain ST8461 into a $14 \mathrm{~mL}$ tube containing $5 \mathrm{~mL} \mathrm{SC}$ medium and incubating at $30^{\circ} \mathrm{C}$ and 250 rpm overnight. Precultures were then used to inoculate $300 \mu \mathrm{L}$ media in 96-deep-well plates from EnzyScreen at $\mathrm{OD}_{600}=0.1$ in 
duplicate for each different medium and incubated at $30^{\circ} \mathrm{C}$ and $225 \mathrm{rpm}$ for $48 \mathrm{~h}$. Samples for analysis were taken at 24 and $48 \mathrm{~h}$.

\section{LC-MS Analysis for Medium Optimization}

Amounts of total ergothioneine were analyzed my LC-MS in MRM mode. The analysis was performed on a Bruker EVOQ
(QqQ) coupled to UPLC. The LC part of the LC-MS/MS system consisted of a CTC autosampler module, a high pressure mixing pump and a column module (Advance, Bruker, Fremont, CA, USA). The injection volume was $1 \mu \mathrm{L}$. The chromatography was performed on a ZIC-cHILIC column, $150 \times 2.1 \mathrm{~mm}, 3 \mu \mathrm{m}$ particle size (SeQuant, Merck Millipore), equipped with a $0.5 \mu$

A

AA

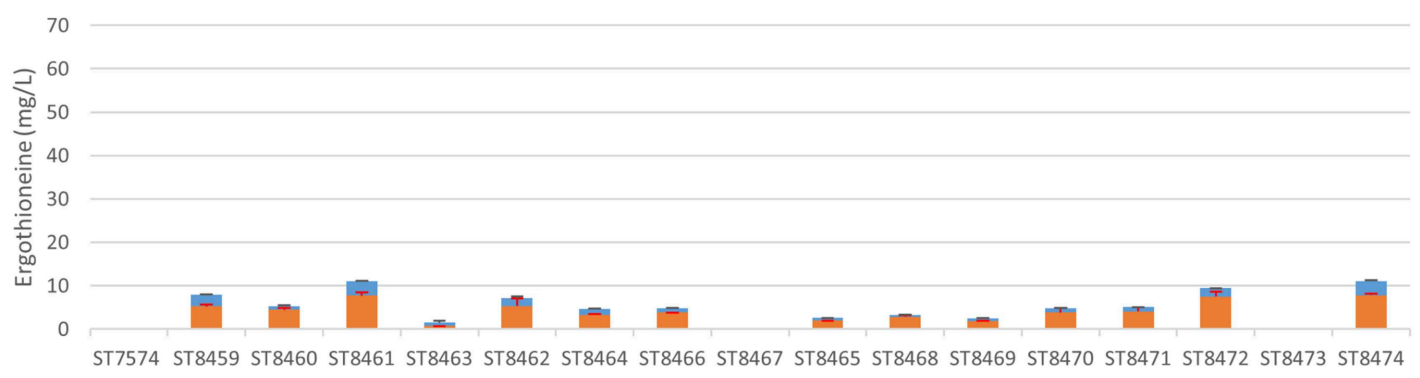

B

Glu

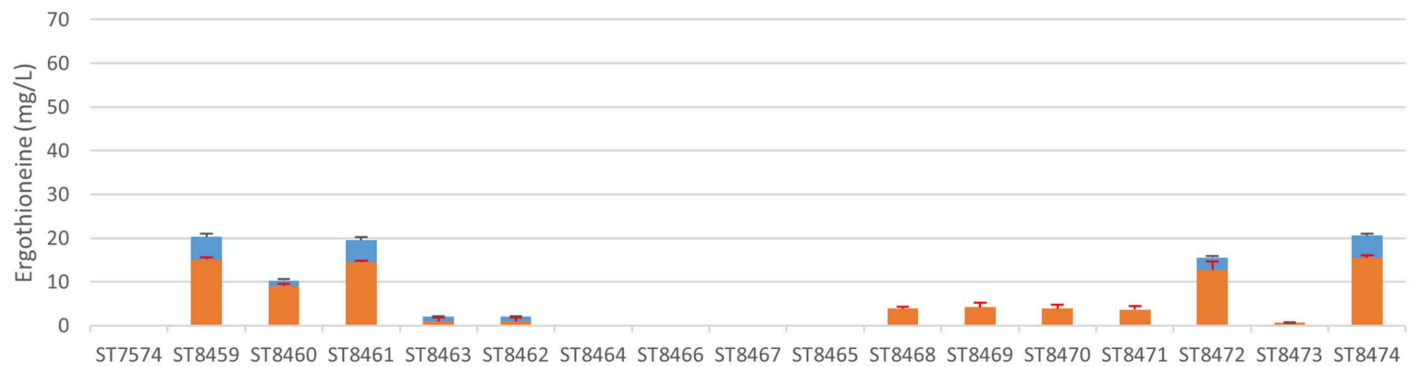

C

FiT

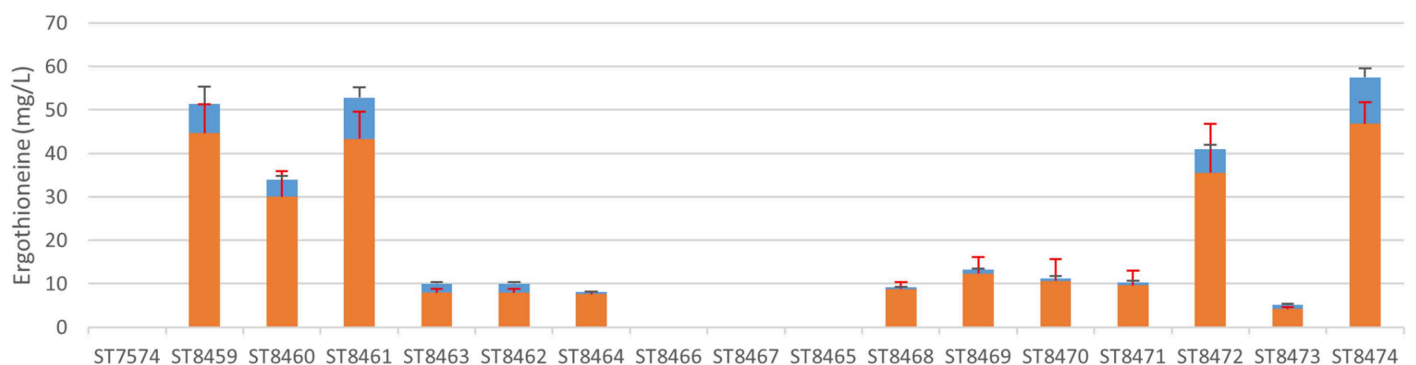

NcEgt1

SpEgt1

CpEgt1

NcEgt2

SpEgt2

CpEgt2

MsEgtA

MsEgtB

MsEgtC

MsEgtD

MsEgtE

ST7574 ST8459 ST8460 ST8461 ST8463 ST8462 ST8464 ST8466 ST8467 ST8465 ST8468 ST8469 ST8470 ST8471 ST8472 ST8473 ST8474
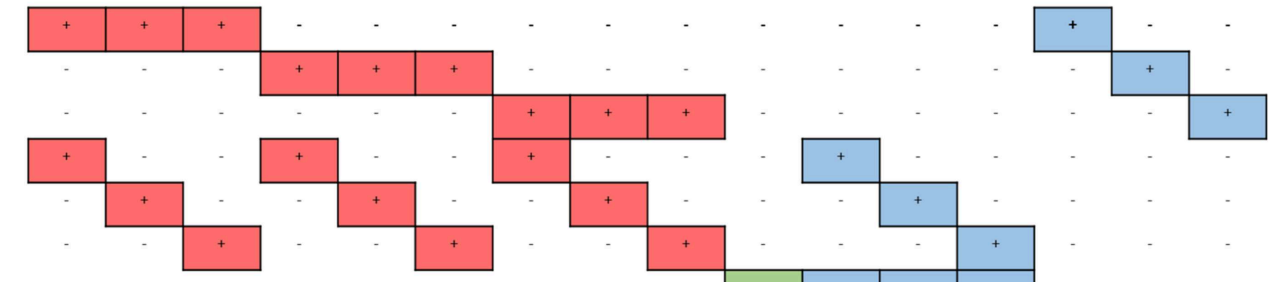

Extracellular

Intracellular

Fungal

Bacterial Mixed

FIGURE 2 | Ergothioneine production in strains with different ERG pathway combinations on three variations of synthetic complete medium: (A) AA: 20 g/l glucose and $1 \mathrm{~g} / \mathrm{l}$ of each L-His, L-Met, and L-Cys, (B) Glu: $40 \mathrm{~g} / \mathrm{l}$ glucose, (C) FiT: $60 \mathrm{~g} / \mathrm{l}$ EnPump substrate for slow glucose release to simulate fed-batch conditions. 
A

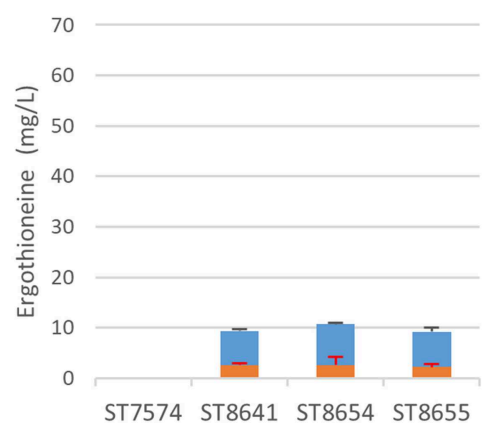

B

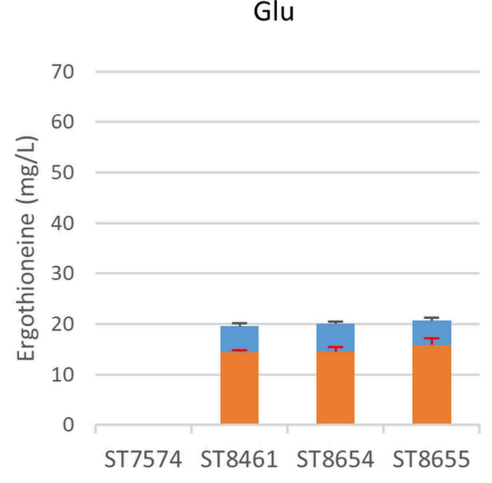

C

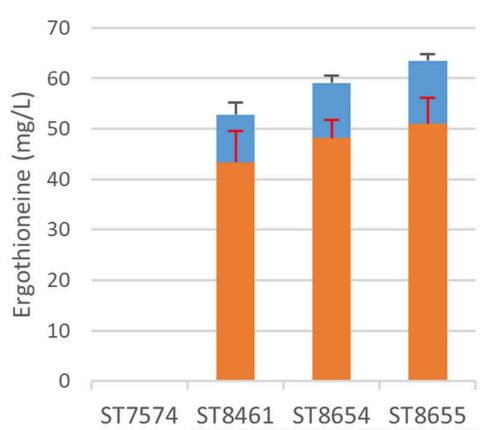

NcEgt1

CpEgt2

MsMEI_6084

Hs.SLC22A4X

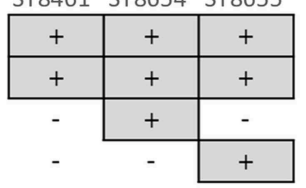

AA

D
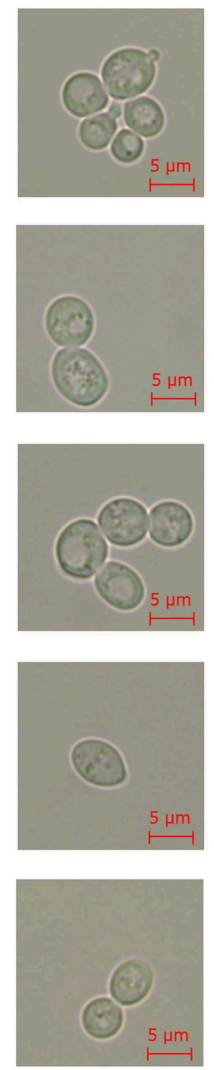

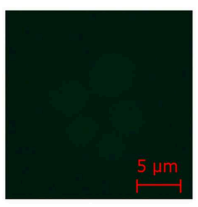

ST8461: Production strain without transporter
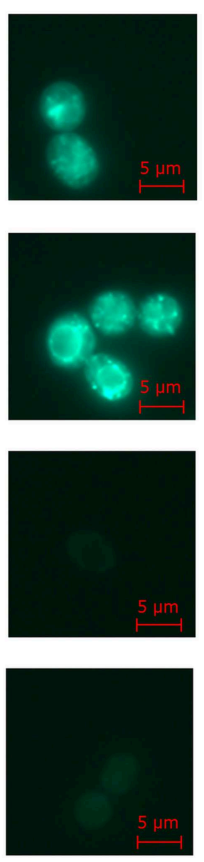

ST8921: Production strain with GFP-MsMEI_6084

ST8922: Production strain with MsMEI_6084-GFP

ST8923: Production strain with HsSLC22A4X-GFP

ST8924: Production strain with GFP-HsSLC22A4X

\section{Extracellular}

FIGURE 3 | Ergothioneine production by yeast strains expressing human ERG transporter SLC22A4X or a putative ERG transporter from M. smegmatis on three variations of synthetic complete medium: (A) AA: 20 g/l glucose and 1 g/l of each L-His, L-Met, and L-Cys, (B) Glu: 40 g/l glucose, (C) FiT: 60 g/l EnPump substrate for slow glucose release to simulate fed-batch conditions, (D) Microscope images of yeast cells with ergothioneine transporters linked to GFP. To the left are Brightfield images and to the right GFP images. Top panels; ST8461, ergothioneine producing strain without transporter. Middle-top panels, ST8921, ergothioneine producing strain with a putative transporter from Mycobacterium smegmatis linked to GFP at the N-terminus. Middle panels, ST8922, ergothioneine producing strain with a putative transporter from Mycobacterium smegmatis linked to GFP at the C-terminus. Middle-bottom panels, ST8923, ergothioneine producing strain with a transporter from Homo sapiens linked to GFP at the C-terminus. Bottom panels, ST8924, ergothioneine producing strain with a transporter from Homo sapiens linked to GFP at the N-terminus.

depth filter (KrudKatcher Classic, Phenomenex). Eluent A was $20 \mathrm{mM}$ ammonium acetate, $\mathrm{pH}$ adjusted to 3.5 with formic acid in MilliQ water. Eluent B was acetonitrile. The total flow rate of eluent $A$ and $B$ was $0.4 \mathrm{~mL} / \mathrm{min}$. The isocratic elution was $30 \% \mathrm{~A}$, and the total run time was $5 \mathrm{~min}$. Retention time was $1.8 \mathrm{~min}$ for ergothioneine. The mass spectrometer was operated 
with electrospray in the positive ion mode (ESI+). The spray voltage was set to $4,500 \mathrm{~V}$. The cone gas flow was $20 \mathrm{~L} / \mathrm{h}$, and the cone temperature was set at $350^{\circ} \mathrm{C}$. The heated probe gas flow was set at $50 \mathrm{~L} / \mathrm{h}$ with a temperature of $350^{\circ} \mathrm{C}$. Nebulizer flow was set at $50 \mathrm{~L} / \mathrm{h}$, and the exhaust gas was turned on. Argon was used as collision gas at a pressure of $1.5 \mathrm{mT}$ Torr. Detection was performed in multiple reacting monitoring (MRM) mode. The quantitative transition was $198 \rightarrow 95$ for ergothioneine and the qualitative transition was $198 \rightarrow 154$. The collision energy was optimized to 15 and $7 \mathrm{eV}$, respectively. The $\mathrm{xms}$ files were converted into cdf files and were analyzed using Mzmine 2.33.

\section{RESULTS}

\section{Expression of Bacterial, Fungal, and Chimeric Biosynthetic Pathways Toward Ergothioneine in S. cerevisiae}

The biosynthetic genes for ERG production were of both bacterial origin (M. smegmatis) and of fungal origin (C. purpurea, $N$. crassa, S. pombe). The Egt1 homologs in C. purpurea and $S$. pombe were identified by BLASTp using the sequence of Egt1 for N. crassa (Genbank accession: XP_956324.3). Similarly, Egt2 from S. pombe (Genbank accession: NP_595091.1) was used to find the Egt2 homologs in N. crassa and C. purpurea. Genbank accession numbers are provided in Supplementary Table 1. The genes were combined into sixteen pathway variants, where nine pathway variants were made of fungal genes, one pathway variant comprised bacterial genes only, and six variants contained both fungal and bacterial genes. The 16 yeast strains with different pathway variants were cultivated in three different media and the intra- and extracellular concentrations of ergothioneine were measured (Figure 2).

ERG titers were the highest in simulated fed-batch medium (up to $60 \mathrm{mg} / \mathrm{L}$ as compared to a maximum of $20 \mathrm{mg} / \mathrm{L}$ under batch conditions). Of the five best-performing pathway variants, four contained Egt1 from $N$. crassa and any other of the four enzymes catalyzing the 2nd enzymatic step (MsEgtE, NcEgt2, SpEgt2, or CpEgt2). The fifth contained CpEgt1 and MsEgtE. Curiously, CpEgt 1 combined with any fungal Egt 2 variants only produced ERG on the medium that contained high levels of histidine, cysteine and methionine (Figure 2A). We speculate that C. purpurea enzyme CpEgt1 has a lower affinity to one or several of the substrate(s) (histidine, SAM, cysteine) than the enzyme from $N$. crassa and hence ERG production was only feasible when the intracellular levels of the substrates was increased through supplementation in the medium. Overall, only $5-30 \%$ of the total ERG was secreted, while the rest was retained intracellularly. The strain ST8461, combining Egt1 from N. crassa and Egt2 from C. purpurea, was selected for further engineering.

\section{Engineering Ergothioneine Transport}

The concentration of ERG inside the cells can be estimated to be $\sim 3.5 \mathrm{mM}$ (for strain ST8461 on simulated fed-batch medium), which is 80 -fold higher than in the broth. As $M$. smegmatis is known to secrete ergothioneine to levels up to 4 times the intracellular concentration (Sao Emani et al.,
2013), we speculated that it may contain an equilibrative or effluxing ERG transporter. On the $M$. smegmatis genome, adjacent to the egtA-E operon, there is an open reading frame MSMEI_6084, encoding a protein annotated as a chloramphenicol exporter (Supplementary Figure 1). The protein has 12 transmembrane domains as predicted by Phyre2 (Kelley et al., 2015) (Supplementary Figure 2). We expressed codon-optimized variants of this gene and of the (normally concentrative) human ERG transporter SLC22A4 in strain ST8461 (Figure 3). However, there was no significant increase of intracellular or extracellular ERG production. To determine why neither transporter had an effect, we investigated their cellular localization by green fluorescent protein (GFP) tagging on the

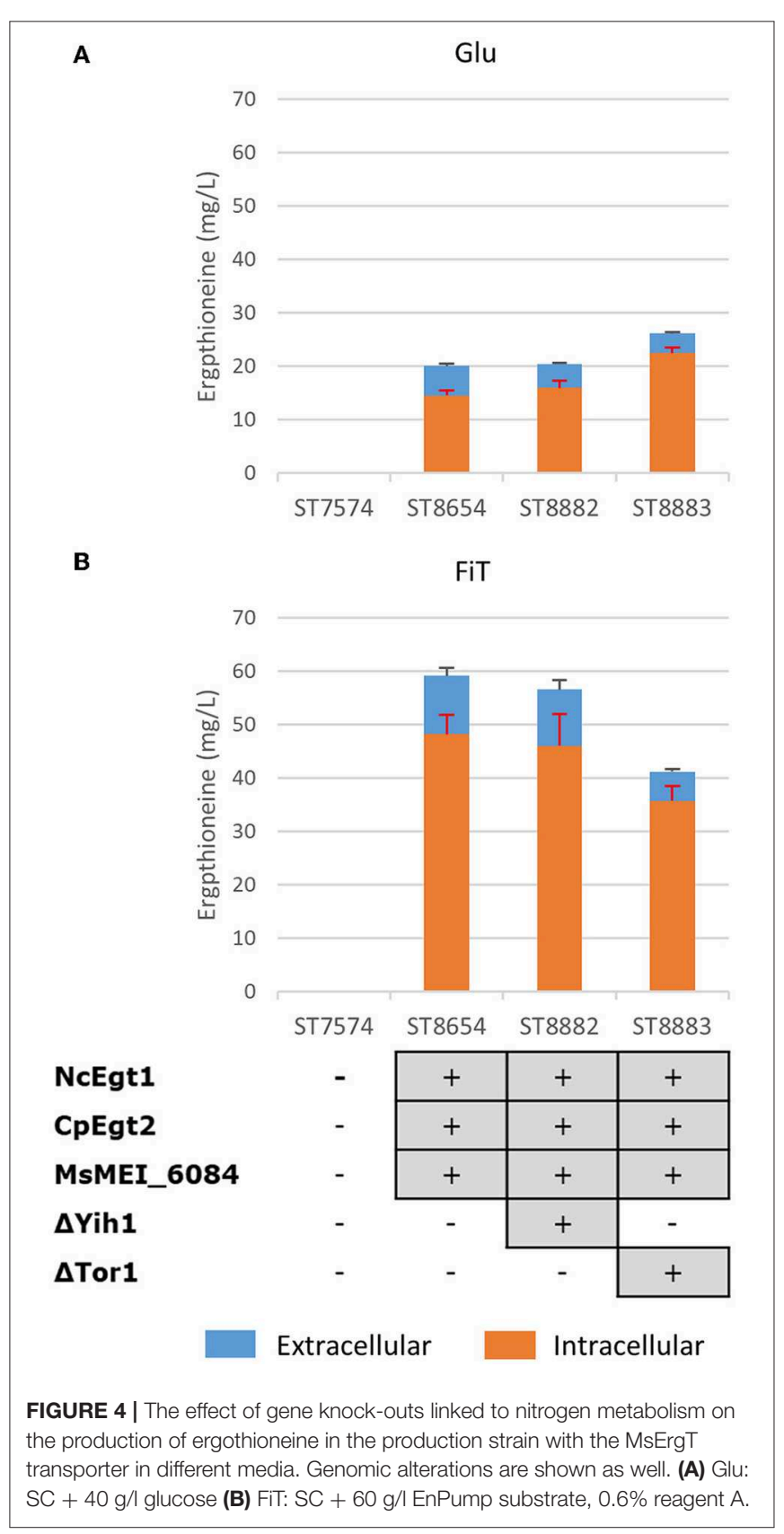


C- or N-termini (Figure 3). Interestingly, MsMEI_6084 mainly localized to the vacuolar membrane of $S$. cerevisiae, while human SLC22A4X was only weakly expressed and its localization could not be determined. Since neither of the transporters localized to the plasma membrane specifically, it can explain the lack of effect on ERG secretion.

\section{Engineering of Nitrogen Metabolism}

Both Tor1 (Hinnebusch, 2005; Ljungdahl and Daignan-Fornier, 2012) and Yih1 (Hinnebusch, 2005) inhibit Gcn2p, a positive regulator of GCN4. Deletion of either of these enzymes could lead to increased ERG production by increasing the amino acid pools in yeast. In Figure 4, we show the results of these alterations in the nitrogen metabolism of $S$. cerevisiae in different media. Yih1 deletion does not have an effect on the production of ergothioneine, while Tor1 only seems to lead to an increase in ERG under batch conditions. Since eventual ERG production is likely to be carried out under fed-batch conditions and neither of the deletions gave a positive effect under fed-batch conditions, we decided not to proceed with these genetic modifications.

\section{Supplementation of the Medium With Precursor Amino Acids}

To determine whether the supply of the three amino acids ( $\mathrm{L}$ histidine, L-cysteine, and L-methionine) that serve as precursors for ERG biosynthesis is limited, we cultivated several yeast strains with supplementation of 1 or $2 \mathrm{~g} / \mathrm{L}$ of each of L-methionine, L-cysteine, and L-histidine. We chose two producing strains, ST8461 and ST8654, the latter containing the MsMEI_8064 gene.

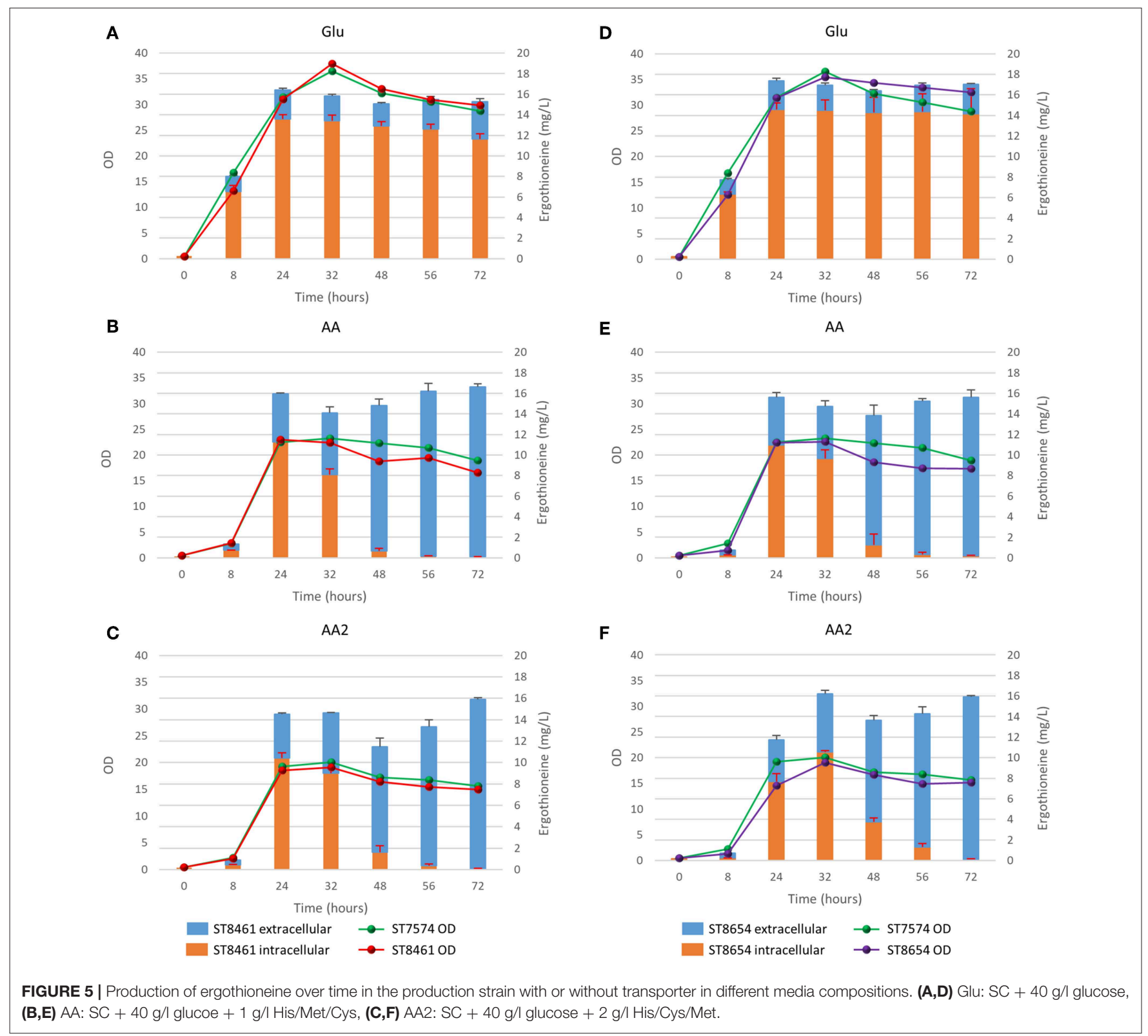


A non-producing strain ST7574 was used as a control. The experiments were performed in shake flasks and growth and ERG production were monitored over the course of 3 days (Figure 5). ERG accumulated primarily in the first $24 \mathrm{~h}$ of cultivation, which would correspond to the exponential growth on glucose, reaching ca. $16 \mathrm{mg} / \mathrm{L}$ in both producing strains, independent of any amino acid supplementation. The supplementation, however, affected the cellular growth, with the final OD being $\sim 46$ and $52 \%$ lower when 1 or $2 \mathrm{~g} / \mathrm{L}$, respectively, of the amino acids were added. No degradation of ERG was observed; however surprisingly, there was a large variation in the intracellular vs. extracellular distribution of ERG depending on the addition of amino acids. Specifically, the addition of amino acids promoted the excretion of ERG in the stationary phase. We hypothesized that this was due to cell death because of the toxic effects of the added amino acids, in particular histidine (Watanabe et al., 2014) and cysteine (Kumar et al., 2006). Indeed propidium iodide staining of cells sampled every $24 \mathrm{~h}$ for $72 \mathrm{~h}$, showed an increase in the fraction of dead cells from 9 to $70 \%$, when amino acids were added at concentrations of $1 \mathrm{~g} / \mathrm{L}$ (Supplementary Figure 3). Clearly, the concentrations of amino acids used were too high and hence we decided to undertake a more systematic medium optimization approach as described in the next section.

\section{Medium Optimization}

To perform optimization of medium composition, we chose a two-level fractional factorial design, where the concentrations each of the components of the synthetic complete medium were varied 5-fold (Figure 6, Supplementary Table 8). Strain ST8461 was cultured in SC medium (medium 65 in Figure 6) to provide a baseline for the ergothioneine production during the experiment with which to compare the performance of the other media. After $48 \mathrm{~h}, 63 \%$ of the designed media outperformed SC medium with regard to ERG titers. To be able to identify the best contributing components, the analysis was narrowed down to the eight top-performing media. Higher levels of arginine, histidine, methionine, and pyridoxine were present in these media, while we could find no compound that had its concentration reduced across most or all of these media. Even though cysteine is a precursor for ergothioneine, it is not universally increased across the different media. However, as methionine can be converted into both S-adenosyl methionine and cysteine by yeast, increased levels of methionine in the medium by itself could be enough for increasing ERG titers. Pyridoxine is a precursor for pyridoxal 5' phosphate (PLP), which binds to EgtE to facilitate the conversion of HCO to 2-(hydroxysulfanyl)hercynine in a PLP-dependent manner (Song et al., 2015). Since CpEgt2 is the fungal equivalent of EgtE, it is likely that pyridoxine has a positive effect on ERG production through $\mathrm{CpEgt} 2$. Interestingly, higher concentrations of arginine also increased ergothioneine production. The amino acid metabolism of $S$. cerevisiae contains many degradation and bioconversion pathways for arginine to be converted into other amino acids (Ljungdahl and Daignan-Fornier, 2012), which might contribute to the increased ERG titers.

\section{Enhancing the Expression of Ergothioneine Biosynthetic Genes}

We recognized that the ERG-producing strain could be improved by increasing the expression of the ERG biosynthetic genes. We integrated an additional copy of NcEgt1 and/or CpEgt2 expression cosseted into ST8461 (Figure 7). An additional copy of NcEgt1 increased the titer by 80 and $20 \%$ for batch and

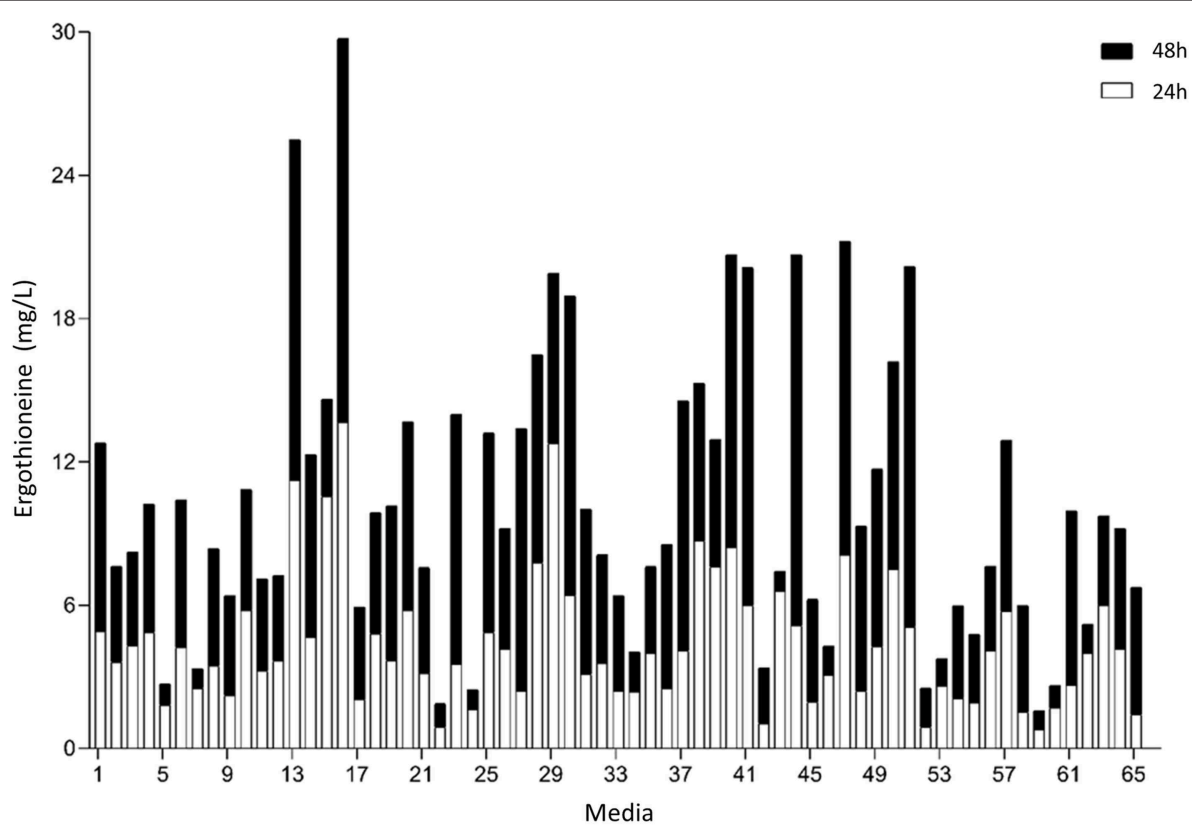

FIGURE 6 | Median concentration of ergothionine produced after 24 and $48 \mathrm{~h}$. The values are additive. Open bars represent values obtained after $24 \mathrm{~h}$ while the closed bars represent values obtained after $48 \mathrm{~h}$. 
A

\section{Glu}

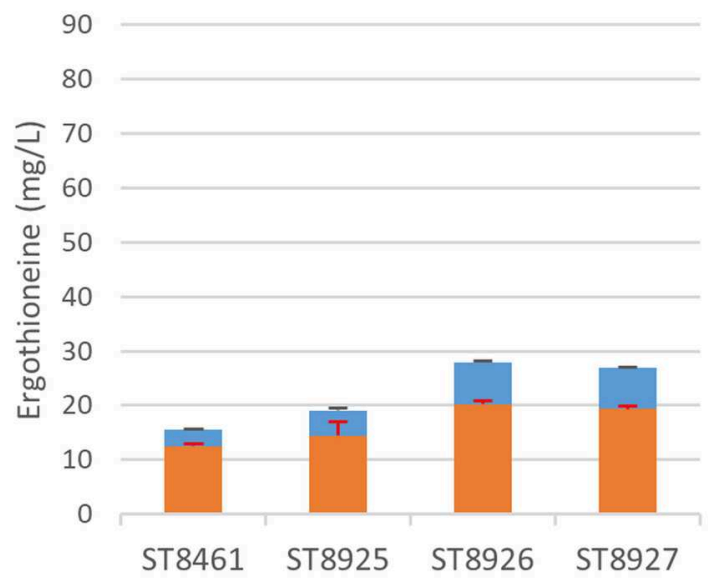

B

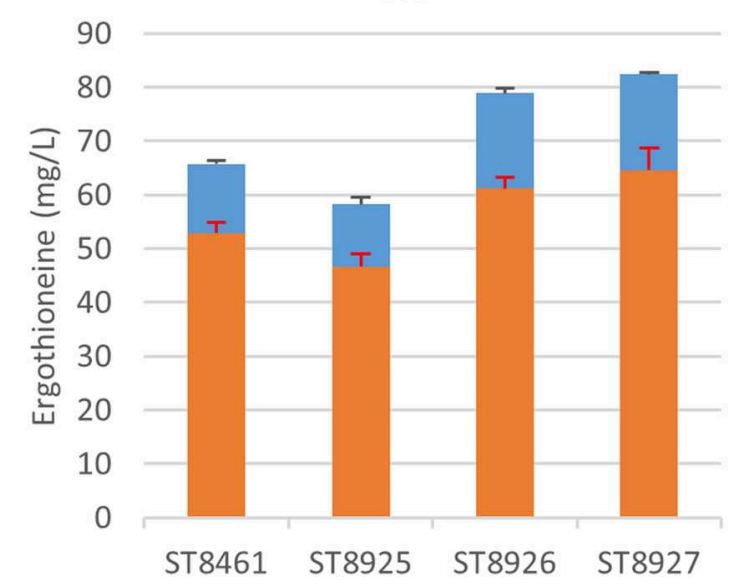

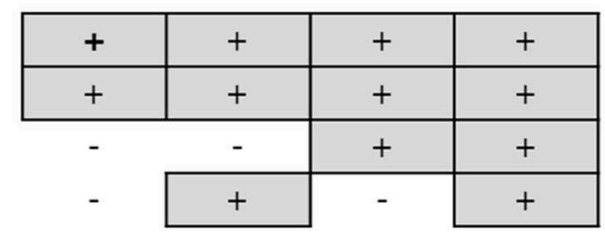

\section{Extracellular}

FIGURE 7 | The effect of the integration of a second copy of Egt enzymes on the production of ergothioneine in the high producing strain in different media. Genomic alterations are shown as well. (A) Glu: SC +40 g/l glucose (B) FiT: $\mathrm{SC}+60 \mathrm{~g} / \mathrm{l}$ EnPump substrate, $0.6 \%$ reagent $\mathrm{A}$.

simulated fed-batch medium, respectively, while an additional copy of Egt 2 did not increase the ergothioneine titer and even caused a decrease in simulated fed-batch medium. When additional copies of both genes were integrated, the total titer was increased by $25 \%$ on simulated fed-batch medium (ST8927). The marginal increase in titer indicates that the flux control of the pathway mainly resides elsewhere, either with the precursor supply or NcEgt1 and CpEgt2 may experience inhibition from
ERG or its intermediates; this needs to be addressed through further strain engineering.

\section{Ergothioneine Production in Controlled Fed-Batch Fermentation}

Finally, the engineered ERG-producing ST8927 strain was cultivated in bioreactors under glucose-limited fed-batch conditions. Based on the medium optimization results from section Medium Optimization, we supplemented the fed-batch fermentation medium with arginine, histidine, methionine and pyridoxine. During the 84 -h cultivation, $598 \pm 18 \mathrm{mg} / \mathrm{L}$ erg was produced, of which 59\% was extracellular, from $175.0 \pm$ $3.5 \mathrm{~g} / \mathrm{L}$ glucose (Figure 8). Next to that, a total of $3.2 \mathrm{~g}$ arginine, histidine, and methionine, as well as $192 \mathrm{mg}$ pyridoxine was added through the starting medium and the feeding medium. The final dry weight of biomass was $55 \pm 1 \mathrm{~g} / \mathrm{L}$, and as baker's yeast has a cell density of $\sim 1.103 \mathrm{~g} / \mathrm{mL}$ (Bryan et al., 2010), this brings the intracellular concentration of ERG to $17.7 \mathrm{mM}$, which is 11-fold higher than the extracellular concentration of $1.6 \mathrm{mM}$ at the end of the fermentation. During the fermentation, at two points (after 48 and $72 \mathrm{~h}$ ), the growth of the cells started stagnating. However, when we added extra $\left(\mathrm{NH}_{4}\right)_{2} \mathrm{SO}_{4}, \mathrm{MgSO}_{4}$, trace metal solution, and vitamins, the cells began growing again. Most likely, the strain has an extra requirement for one or more of these components that was not found via the medium optimization, as the medium optimization was run under batch conditions, rather than fed-batch conditions in which yeast can reach much higher cell densities. Between 48 and $60 \mathrm{~h}$, biomass concentration declined slightly and the ratio between the extra- and the intra-cellular ERG increased, which could be caused by increased cell death, similar as we observed upon the supplementation of high concentrations of amino acids (section Medium Optimization). The process performance could be improved by optimization of the medium composition, process physical parameters, and the feeding profile.

\section{DISCUSSION}

Ergothioneine is an antioxidant with many potential health benefits (Cheah and Halliwell, 2012; Ames, 2018; Halliwell et al., 2018). Furthermore, the interaction of ERG with metal ions (Hanlon, 1971) could conceivably play a role in the intracellular chaperoning of trace elements. Fermentation could provide an alternative and sustainable way for ergothioneine production compared to the current commercial processes. To this end, we have engineered $S$. cerevisiae for the production of ergothioneine, reaching levels of $0.6 \mathrm{~g} / \mathrm{L}$. As there are many different organisms that produce ergothioneine (Tanret, 1909; Genghof et al., 1956; Genghof and Vandamme, 1964; Genghof, 1970; Seebeck, 2010; Pfeiffer et al., 2011; Pluskal et al., 2014; Sheridan et al., 2016; Kalaras et al., 2017) (Supplementary Tables 9, 10), we have focused on the organisms in which genes for ergothioneine production were identified. Other heterologous or from different organisms could therefore also be tested in S. cerevisiae to potentially find better performing combinations than those described here. 


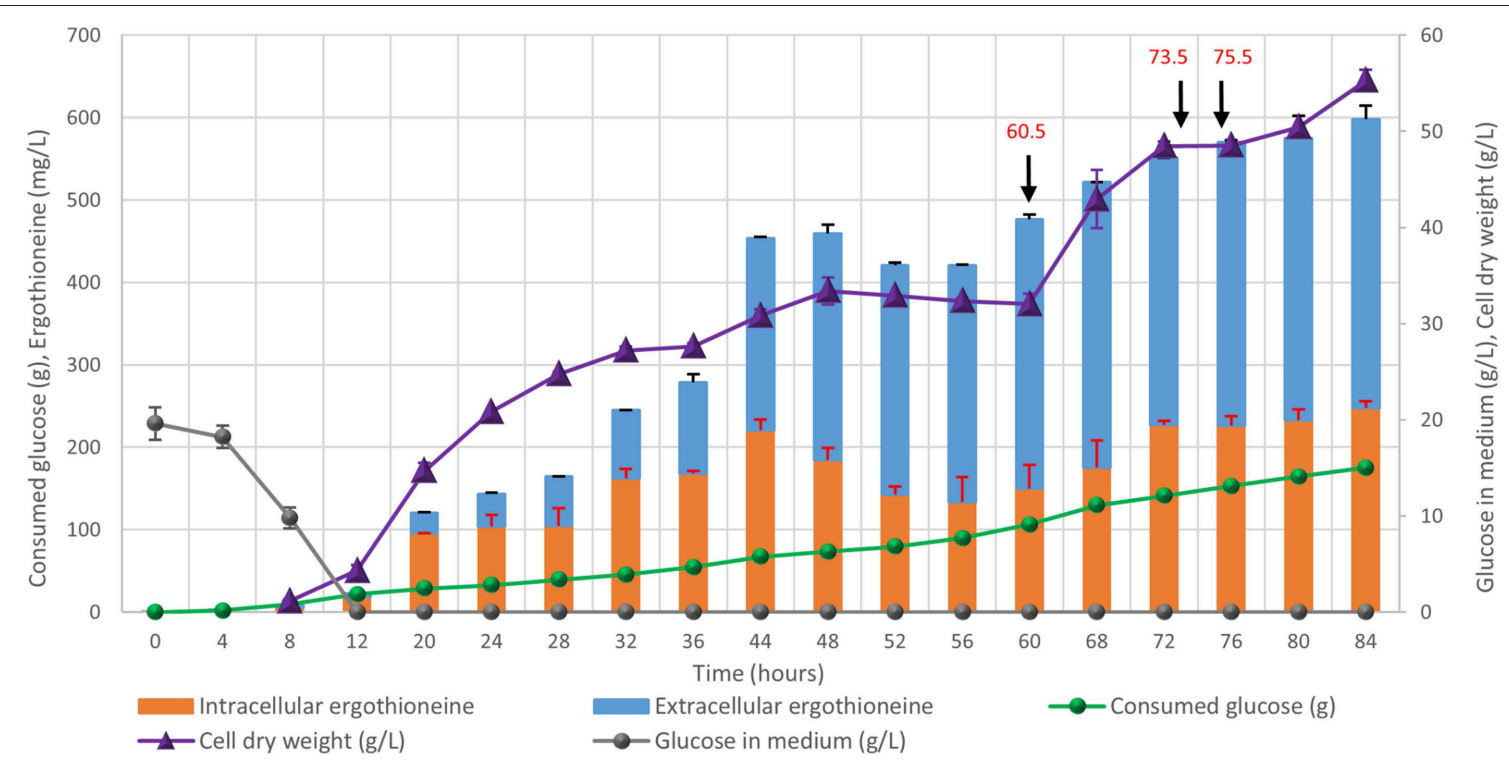

FIGURE 8 | Fed-batch cultivation of ERG-producing strain ST8927. The cultivations were performed in duplicate, the average values are shown. The error bars show

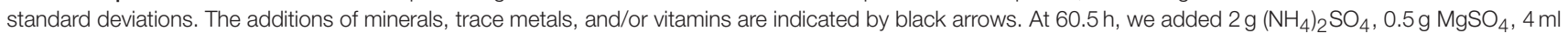
trace metals solution, and $2 \mathrm{ml}$ vitamin solution, at $73.5 \mathrm{~h}$ we added $0.5 \mathrm{~g} \mathrm{MgSO}, 4 \mathrm{ml}$ trace metals solution $2 \mathrm{ml}$ vitamin solution and at $75.5 \mathrm{~h}$ we added $2 \mathrm{~g}$ $\left(\mathrm{NH}_{4}\right)_{2} \mathrm{SO}_{4}$. At 60.5 and $75.5 \mathrm{~h}, 2 \mathrm{~g}\left(\mathrm{NH}_{4}\right)_{2} \mathrm{SO}_{4}$ was added as a sterile $100 \mathrm{~g} / \mathrm{l}$ solution. At 60.5 and $73.5 \mathrm{~h}, 0.5 \mathrm{~g} \mathrm{MgSO} 4$ was added as a sterile $50 \mathrm{~g} / \mathrm{l}$ solution, while $4 \mathrm{ml}$ sterile trace metals solution and $2 \mathrm{ml}$ sterile vitamin solution were added.

In our ERG-producing strain, as much as 59\% of the ergothioneine was detected extracellularly during fermentation, even though no ERG-specific transporter has been found in yeast so far. High intracellular concentrations of a product may inhibit its own biosynthesis or lead to product degradation (Kell et al., 2015; Borodina, 2019; Kell, 2019). We speculated that expressing an ERG-specific transporter might improve the secretion of ERG from the yeast cells. Expression of heterologous human ergothioneine transporter or a potential ergothioneine transporter from $M$. smegmatis did not improve the secretion, however the transporters were not well-expressed in the plasma membrane of $S$. cerevisiae.

The amino acid metabolism of $S$. cerevisiae is tightly regulated and its networks highly intertwined (Hinnebusch, 1988, 2005; Hinnebusch and Natarajan, 2002; Ljungdahl and DaignanFornier, 2012). Therefore, it would be easier to increase the total amino acid pool rather than increasing the individual amino acid pools. The general amino acid control of yeast is mainly regulated by GCN4 (Hinnebusch, 1988; Ljungdahl and DaignanFornier, 2012) and upregulation of GCN4 leads to transcription of the biosynthetic genes of various amino acids. As knockout of Tor1 and Yih1 did not yield higher ERG titers, more difficult metabolic engineering of yeast for higher production of individual amino acids pools could lead to higher ERG titers. This is similar to the approach taken in E. coli, where use was made of a strain that was already overproducing cysteine (Osawa et al., 2018). In yeast, it is also possible to adopt several strategies that increase the amount of available SAM and/or methionine (Chen et al., 2016) to potentially improve ergothioneine production.
Additional to genetic manipulation, medium optimization has the benefit that it does not require extensive engineering of the strain to increase its production capabilities, while it gives a wealth of information on multiple components in the system that the strain might need for better production (Link and Weuster-Botz, 2011). However, supplementation of certain compounds can be expensive in an industrial setting and therefore the information gained can be used to lead further engineering efforts.

Ergothioneine production was only increased by a small amount following the integration of a second copy of both Egt genes. There are a number of potential explanations for this, such as a lack of precursors or [as is common (Cornishbowden et al., 1995)] possible feedback inhibition by pathway intermediates or products. Interestingly, integrating a second copy of NcEgt1 by itself, but not CpEgt2, did lead to an increase in ERG production. Indeed, in engineering $A$. oryzae (Takusagawa et al., 2019), it has been shown that hercynine accumulated following the integration of multiple copies of Egt1. This suggests that the second reaction catalyzed by the Egtl enzyme, converting hercynine into $\mathrm{HCO}$, tends to contribute more significantly to flux control.

Recently, another group managed to produce $1.3 \mathrm{~g} / \mathrm{L}$ ergothioneine in using $E$. coli as a production organism (Tanaka et al., 2019). They used a cysteine hyperproducing strain, which was also engineered for increased methionine production. Additionally, their ERG production genes are on a plasmid which is present in the cells with 15-20 copy numbers. During fermentation, they supplemented the medium with histidine, methionine, thiosulfate, and pyridoxine, supporting the results 
found in our medium optimization experiment. While the titer obtained in E. coli was higher than in our fed-batch fermentation, the duration of the fermentation with $E$. coli was much longer at $216 \mathrm{~h}$ vs. the $84 \mathrm{~h}$-fermentation using $S$. cerevisae presented here.

The fungal pathway for the biosynthesis of ERG only encompasses two enzymes compared to the five of the bacterial pathway, and eliminates the need for the use of glutamate and energy in the form of ATP. While NcEgt1 has been produced in $E$. coli for in vitro studies of the enzyme (Hu et al., 2014), to the best of our knowledge, the fungal pathway has to date not been used for ERG production in E. coli. As we have shown S. cerevisiae is able to produce ergothioneine using the fungal pathway, the more energetically efficient biosynthesis pathway of fungi could lead to better product yield.

In conclusion, we produced ERG with a titer of $598 \pm 18 \mathrm{mg} / \mathrm{L}$ in $S$. cerevisiae expressing fungal ERG biosynthesis pathway. As the first report of ergothioneine production in S. cerevisiae, our investigation into the impact of amino acid supplementation and the flux in the ERG pathway will help further ERG production efforts by yeast fermentation.

\section{DATA AVAILABILITY STATEMENT}

All datasets generated for this study are included in the manuscript/Supplementary Files.

\section{AUTHOR CONTRIBUTIONS}

IB and DK conceived the study. IB, SH, BP, and BD designed the experiments and analyzed the data. $\mathrm{SH}$ and $\mathrm{KZ}$ performed

\section{REFERENCES}

Akanmu, D., Cecchini, R., Aruoma, O. I., and Halliwell, B. (1991). The antioxidant action of ergothioneine. Arch. Biochem. Biophys. 288, 10-16. doi: 10.1016/0003-9861(91)90158-F

Alamgir, K. M., Masuda, S., Fujitani, Y., Fukuda, F., and Tani, A. (2015). Production of ergothioneine by Methylobacterium species. Front. Microbiol. 6:1185. doi: $10.3389 /$ fmicb.2015.01185

Ames, B. N. (2018). Prolonging healthy aging: longevity vitamins and proteins. Proc. Natl. Acad. Sci. U.S.A. 115, 10836-10844. doi: 10.1073/pnas.1809045115

Audley, B. G., and Tan, C. H. (1968). The uptake of ergothioneine from the soil into the latex of Hevea brasiliensis. Phytochemistry 7, 1999-2000. doi: 10.1016/S0031-9422(00)90759-3

Barger, G., and Ewins, A. J. (1911). CCLVII.-the constitution of ergothioneine: a betaine related to histidine. J. Chem. Soc. Trans. 99, 2336-2341. doi: 10.1039/CT9119902336

Bedirli, A., Sakrak, O., Muhtaroglu, S., Soyuer, I., Guler, I., Riza Erdogan, A., et al. (2004). Ergothioneine pretreatment protects the liver from ischemiareperfusion injury caused by increasing hepatic heat shock protein 70. J. Surg. Res. 122, 96-102. doi: 10.1016/j.jss.2004.06.016

Borodina, I. (2019). Understanding metabolite transport gives an upper hand in strain development. Microb. Biotechnol. 12, 69-70. doi: 10.1111/1751-7915.13347

Borodina, I., Kildegaard, K. R., Jensen, N. B., Blicher, T. H., Maury, J., Sherstyk, S., et al. (2015). Establishing a synthetic pathway for highlevel production of 3-hydroxypropionic acid in Saccharomyces cerevisiae via $\beta$-alanine. Metab. Eng. 27, 57-64. doi: 10.1016/j.ymben.2014. 10.003 the strain screening and transporter experiments. MB performed the nitrogen metabolism alteration experiments. SH performed the amino acid supplementation time course. BP performed the medium optimization. The second copy integration experiments were performed by $\mathrm{SH}$. The fed-batch fermentation was performed by SH, MR, and JM. SH, IB, and DK wrote the manuscript. IB and DK secured the funding and supervised the project.

\section{FUNDING}

This project has received funding from the European Research Council (ERC) under the European Union's Horizon 2020 research and innovation programme (grant agreement No 757384). The work has also been funded by the Novo Nordisk Foundation (grant number NNF10CC1016517). Furthermore, funding from an Erasmus+ grant (grant agreement no. 20171-PL01-KA103-035590, university code PL WROCLAW04) has contributed to this work.

\section{ACKNOWLEDGMENTS}

The authors would like to thank Jolanda ter Horst for her help with the fed-batch fermentation experiment.

\section{SUPPLEMENTARY MATERIAL}

The Supplementary Material for this article can be found online at: https://www.frontiersin.org/articles/10.3389/fbioe. 2019.00262/full\#supplementary-material
Bryan, A. K., Goranov, A., Amon, A., and Manalis, S. R. (2010). Measurement of mass, density, and volume during the cell cycle of yeast. Proc. Natl. Acad. Sci. U.S.A. 107, 999-1004. doi: 10.1073/pnas.0901851107

Burn, R., Misson, L., Meury, M., and Seebeck, F. P. (2017). Anaerobic origin of ergothioneine. Angew. Chem. Int. Ed. 56, 12508-12511. doi: 10.1002/anie.201705932

Cheah, I. K., and Halliwell, B. (2012). Ergothioneine; antioxidant potential, physiological function and role in disease. Biochim. Biophys. Acta 1822, 784-793. doi: 10.1016/j.bbadis.2011.09.017

Cheah, I. K., Tang, R., Ye, P., Yew, T. S. Z., Lim, K. H. C., and Halliwell, B. (2016). Liver ergothioneine accumulation in a guinea pig model of non-alcoholic fatty liver disease. A possible mechanism of defence? Free Radic. Res. 50, 14-25. doi: 10.3109/10715762.2015.1099642

Cheah, I. K., Tang, R. M. Y., Yew, T. S. Z., Lim, K. H. C., and Halliwell, B. (2017). Administration of pure ergothioneine to healthy human subjects: uptake, metabolism, and effects on biomarkers of oxidative damage and inflammation. Antioxid. Redox Signal. 26, 193-206. doi: 10.1089/ars.201 6.6778

Chen, H., Wang, Z., Cai, H., and Zhou, C. (2016). Progress in the microbial production of S-adenosyl-l-methionine. World J. Microbiol. Biotechnol. 32:153. doi: 10.1007/s11274-016-2102-8

Cornishbowden, A., Hofmeyr, J. H. S., and Cardenas, M. L. (1995). Strategies for manipulating metabolic fluxes in biotechnology. Bioorg. Chem. 23, 439-449. doi: 10.1006/bioo.1995.1030

Deiana, M., Rosa, A., Casu, V., Piga, R., Assunta Desi, M., and Aruoma, O. I. (2004). L-Ergothioneine modulates oxidative damage in the kidney and liver of rats in vivo: studies upon the profile of polyunsaturated fatty acids. Clin. Nutr. 23, 183-193. doi: 10.1016/S0261-5614(03)00108-0 
Ey, J., Schömig, E., and Taubert, D. (2007). Dietary sources and antioxidant effects of ergothioneine. J. Agric. Food Chem. 55, 6466-6474. doi: 10.1021/jf07 $1328 \mathrm{f}$

Fujitani, Y., Alamgir, K. M., and Tani, A. (2018). Ergothioneine production using Methylobacterium species, yeast, and fungi. J. Biosci. Bioeng. 126, 715-722. doi: 10.1016/j.jbiosc.2018.05.021

Genghof, D. S. (1970). Biosynthesis of ergothioneine and hercynine by fungi and Actinomycetales. J. Bacteriol. 103, 475-478.

Genghof, D. S., Inamine, E., Kovalenko, V., and Melville, D. B. (1956). Ergothioneine in microorganisms. J. Biol. Chem. 223, 9-17.

Genghof, D. S., and Vandamme, O. (1964). Biosynthesis of ergothioneine and hercynine by mycobacteria. J. Bacteriol. $87,852-862$.

Grundemann, D., Harlfinger, S., Golz, S., Geerts, A., Lazar, A., Berkels, R., et al. (2005). Discovery of the ergothioneine transporter. Proc. Natl. Acad. Sci. U.S.A. 102, 5256-5261. doi: 10.1073/pnas.0408624102

Guo, Q.-L., Lin, S., Wang, Y.-N., Zhu, C.-G., Xu, C.-B., and Shi, J.-G. (2016). Gastrolatathioneine, an unusual ergothioneine derivative from an aqueous extract of "tian ma": a natural product co-produced by plant and symbiotic fungus. Chin. Chem. Lett. 27, 1577-1581. doi: 10.1016/j.cclet.2016. 06.040

Halliwell, B., Cheah, I. K., and Tang, R. M. Y. (2018). Ergothioneine - a dietderived antioxidant with therapeutic potential. FEBS Lett. 592, 3357-3366. doi: 10.1002/1873-3468.13123

Hanlon, D. P. (1971). Interaction of ergothioneine with metal ions and metalloenzymes. J. Med. Chem. 14, 1084-1087. doi: 10.1021/jm00293a017

Hinnebusch, A. G. (1988). Mechanisms of gene regulation in the general control of amino acid biosynthesis in Saccharomyces cerevisiae. Microbiol. Rev. 52, 248-273.

Hinnebusch, A. G. (2005). Translational regulation of GCN4 and the general amino acid control of yeast. Annu. Rev. Microbiol. 59, 407-450. doi: 10.1146/annurev.micro.59.031805.133833

Hinnebusch, A. G., and Natarajan, K. (2002). Gcn4p, a master regulator of gene expression, is controlled at multiple levels by diverse signals of starvation and stress. Eukaryot. Cell 1, 22-32. doi: 10.1128/EC.01.1.22-32.2002

Hu, W., Song, H., Sae Her, A., Bak, D. W., Naowarojna, N., Elliott, S. J., et al. (2014). Bioinformatic and biochemical characterizations of C-S Bond formation and cleavage enzymes in the fungus Neurospora crassa ergothioneine biosynthetic pathway. Organ. Lett. 16, 5382-5385. doi: 10.1021/ol502596z

Huang, B., Guo, J., Yi, B., Yu, X., Sun, L., and Chen, W. (2008). Heterologous production of secondary metabolites as pharmaceuticals in Saccharomyces cerevisiae. Biotechnol. Lett. 30, 1121-1137. doi: 10.1007/s10529-0089663-z

Jessop-Fabre, M. M., Jakočiunas, T., Stovicek, V., Dai, Z., Jensen, M. K., Keasling, J. D., et al. (2016). EasyClone-MarkerFree: a vector toolkit for markerless integration of genes into Saccharomyces cerevisiae via CRISPR-Cas9. Biotechnol. J. 11, 1110-1117. doi: 10.1002/biot.201600147

Kalaras, M. D., Richie, J. P., Calcagnotto, A., and Beelman, R. B. (2017). Mushrooms: a rich source of the antioxidants ergothioneine and glutathione. Food Chem. 233, 429-433. doi: 10.1016/j.foodchem.2017.04.109

Kell, D. B. (2019). "Control of metabolite efflux in microbial cell factories: current advances and future prospects," in Fermentation Microbiology and Biotechnology, eds E. M. T. El-Mansi, J. Nielsen, D. Mousdale, T. Allman, and P. C. Ross (Boca Raton, FL: CRC Press), 117-138.

Kell, D. B., Swainston, N., Pir, P., and Oliver, S. G. (2015). Membrane transporter engineering in industrial biotechnology and whole cell biocatalysis. Trends Biotechnol. 33, 237-246. doi: 10.1016/j.tibtech.2015.02.001

Kelley, L. A., Mezulis, S., Yates, C. M., Wass, M. N., and Sternberg, M. J. E. (2015). The Phyre2 web portal for protein modeling, prediction and analysis. Nat. Protoc. 10, 845-858. doi: 10.1038/nprot.2015.053

Kumar, A., John, L., Alam, M. M., Gupta, A., Sharma, G., Pillai, B., et al. (2006). Homocysteine- and cysteine-mediated growth defect is not associated with induction of oxidative stress response genes in yeast. Biochem. J. 396, 61-69. doi: 10.1042/BJ20051411

Leisinger, F., Burn, R., Meury, M., Lukat, P., and Seebeck, F. P. (2019). Structural and mechanistic basis for anaerobic ergothioneine biosynthesis. J. Am. Chem. Soc. 141, 6906-6914. doi: 10.1021/jacs.8b12596

Leone, E., and Mann, T. (1951). Ergothioneine in the seminal vesicle secretion. Nature 168, 205-206. doi: 10.1038/168205b0
Li, M., and Borodina, I. (2014). Application of synthetic biology for production of chemicals in yeast Saccharomyces cerevisiae. FEMS Yeast Res. 15, 1-12. doi: $10.1111 / 1567-1364.12213$

Link, C. D. (1995). Expression of human beta-amyloid peptide in transgenic Caenorhabditis elegans. Proc. Natl. Acad. Sci. U.S.A. 92, 9368-9372. doi: 10.1073 /pnas.92.20.9368

Link, H., and Weuster-Botz, D. (2011). "Medium formulation and development," in Comprehensive Biotechnology, ed M. Moo-Young (Amsterdam: Elsevier), 119-134. doi: 10.1016/B978-0-08-088504-9.00092-1

Ljungdahl, P. O., and Daignan-Fornier, B. (2012). Regulation of amino acid, nucleotide, and phosphate metabolism in Saccharomyces cerevisiae. Genetics 190, 885-929. doi: 10.1534/genetics.111.133306

Melville, D. B. (1959). Ergothioneine. Vitam. Horm. 17, 155-204 doi: 10.1016/S0083-6729(08)60271-X

Melville, D. B., Horner, W. H., and Lubschez, R. (1954). Tissue ergothioneine. J. Biol. Chem. 206, 221-228.

Osawa, R., Kamide, T., Satoh, Y., Kawano, Y., Ohtsu, I., and Dairi, T. (2018). Heterologous and high production of ergothioneine in Escherichia coli. J. Agric. Food Chem. 66, 1191-1196. doi: 10.1021/acs.jafc.7b04924

Park, E. J., Lee, W. Y., Kim, S. T., Ahn, J. K., and Bae, E. K. (2010). Ergothioneine accumulation in a medicinal plant Gastrodia elata. J. Med. Plants Res. 4, 1141-1147. doi: 10.5897/JMPR10.184

Paul, B. D., and Snyder, S. H. (2010). The unusual amino acid L-ergothioneine is a physiologic cytoprotectant. Cell Death Differ. 17, 1134-1140. doi: $10.1038 /$ cdd. 2009.163

Pfeiffer, C., Bauer, T., Surek, B., Schömig, E., and Gründemann, D. (2011). Cyanobacteria produce high levels of ergothioneine. Food Chem. 129, 1766-1769. doi: 10.1016/j.foodchem.2011.06.047

Pluskal, T., Ueno, M., and Yanagida, M. (2014). Genetic and metabolomic dissection of the ergothioneine and selenoneine biosynthetic pathway in the fission yeast, S. pombe, and construction of an overproduction system. PLoS ONE 9:e97774. doi: 10.1371/journal.pone.0097774

Sakrak, O., Kerem, M., Bedirli, A., Pasaoglu, H., Akyurek, N., Ofluoglu, E., et al. (2008a). Ergothioneine modulates proinflammatory cytokines and heat shock protein 70 in mesenteric ischemia and reperfusion injury. J. Surg. Res. 144, 36-42. doi: 10.1016/j.jss.2007.04.020

Sakrak, Ö., Kerem, M., Bedirli, A., Pasaoglu, H., Alper, M., Ofluoglu, E., et al. (2008b). Ergothioneine prevents acute lung injury in mesenteric ischemia and reperfusion injury in rats. J. Crit. Care 23, 268-269. doi: 10.1016/j.jcrc.2008.03.021

Sao Emani, C., Williams, M. J., Van Helden, P. D., Taylor, M. J. C., Wiid, I. J., and Baker, B. (2018). Gamma-glutamylcysteine protects ergothioneine-deficient Mycobacterium tuberculosis mutants against oxidative and nitrosative stress. Biochem. Biophys. Res. Commun. 495, 174-178. doi: 10.1016/j.bbrc.2017.10.163 Sao Emani, C., Williams, M. J., Wiid, I. J., Hiten, N. F., Viljoen, A. J., Pietersen, R.-D. D., et al. (2013). Ergothioneine is a secreted antioxidant in Mycobacterium smegmatis. Antimicrob. Agents Chemother. 57, 3202-3207. doi: 10.1128/AAC.02572-12

Seebeck, F. P. (2010). In vitro reconstitution of Mycobacterial ergothioneine biosynthesis. J. Am. Chem. Soc. 132, 6632-6633. doi: 10.1021/ja101721e

Sheridan, K. J., Lechner, B. E., Keeffe, G. O., Keller, M. A., Werner, E. R., Lindner, H., et al. (2016). Ergothioneine biosynthesis and functionality in the opportunistic fungal pathogen, Aspergillus fumigatus. Sci. Rep. 6:35306. doi: $10.1038 /$ srep35306

Shires, T. K., Brummel, M. C., Pulido, J. S., and Stegink, L. D. (1997) Ergothioneine distribution in bovine and porcine ocular tissues. Comp. Biochem. Physiol. C. Pharmacol. Toxicol. Endocrinol. 117, 117-120. doi: 10.1016/S0742-8413(96)00223-X

Song, H., Hu, W., Naowarojna, N., Her, A. S., Wang, S., Desai, R., et al. (2015). Mechanistic studies of a novel C-S lyase in ergothioneine biosynthesis: the involvement of a sulfenic acid intermediate. Sci. Rep. 5:11870. doi: 10.1038/srep11870

Song, T.-Y., Lin, H.-C., Chen, C.-L., Wu, J.-H., Liao, J.-W., and Hu, M.-L. (2014). Ergothioneine and melatonin attenuate oxidative stress and protect against learning and memory deficits in C57BL/6J mice treated with D-galactose. Free Radic. Res. 48, 1049-1060. doi: 10.3109/10715762.2014.920954

Stovicek, V., Borodina, I., and Forster, J. (2015). CRISPR-Cas system enables fast and simple genome editing of industrial Saccharomyces cerevisiae 
strains. Metab. Eng. Commun. 2, 13-22. doi: 10.1016/j.meteno.2015. 03.001

Swainston, N., Currin, A., Day, P. J., and Kell, D. B. (2014). GeneGenie: optimized oligomer design for directed evolution. Nucleic Acids Res. 42, W395-W400. doi: 10.1093/nar/gku336

Ta, P., Buchmeier, N., Newton, G. L., Rawat, M., and Fahey, R. C. (2011). Organic hydroperoxide resistance protein and ergothioneine compensate for loss of mycothiol in Mycobacterium smegmatis mutants. J. Bacteriol. 193, 1981-1990. doi: 10.1128/JB.01402-10

Takusagawa, S., Satoh, Y., Ohtsu, I., and Dairi, T. (2019). Ergothioneine production with Aspergillus oryzae. Biosci. Biotechnol. Biochem. 83, 181-184. doi: 10.1080/09168451.2018.1527210

Tan, C., and Audley, B. (1968). Ergothioneine and hercynine in Hevea brasiliensis latex. Phytochemistry 7, 109-118. doi: 10.1016/S0031-9422(00) 88213-8

Tanaka, N., Kawano, Y., Satoh, Y., Dairi, T., and Ohtsu, I. (2019). Gramscale fermentative production of ergothioneine driven by overproduction of cysteine in Escherichia coli. Sci. Rep. 9:1895. doi: 10.1038/s41598-018-3 8382-w

Tang, Y., Masuo, Y., Sakai, Y., Wakayama, T., Sugiura, T., Harada, R., et al. (2016). Localization of xenobiotic transporter OCTN1/SLC22A4 in hepatic stellate cells and its protective role in liver fibrosis. J. Pharm. Sci. 105, 1779-1789. doi: 10.1016/j.xphs.2016.02.023

Tanret, C. (1909). The new base drawn from rye ergot, ergothioneine. C. R. Hebd. Acad. Sci. 25, 222-224. doi: 10.1080/003692209087 33970

Tschirka, J., Kreisor, M., Betz, J., and Gründemann, D. (2018). Substrate selectivity check of the ergothioneine transporter. Drug Metab. Dispos. 46, 779-785. doi: $10.1124 / \mathrm{dmd} .118 .080440$
Wang, J., Guleria, S., Koffas, M. A., and Yan, Y. (2016). Microbial production of value-added nutraceuticals. Curr. Opin. Biotechnol. 37, 97-104. doi: 10.1016/j.copbio.2015.11.003

Watanabe, D., Kikushima, R., Aitoku, M., Nishimura, A., Ohtsu, I., Nasuno, R., et al. (2014). Exogenous addition of histidine reduces copper availability in the yeast Saccharomyces cerevisiae. Microb. Cell 1, 241-246. doi: 10.15698/mic2014.07.154

Yang, N.-C., Lin, H.-C., Wu, J.-H., Ou, H.-C., Chai, Y.-C., Tseng, C.-Y., et al. (2012). Ergothioneine protects against neuronal injury induced by $\beta$-amyloid in mice. Food Chem. Toxicol. 50, 3902-3911. doi: 10.1016/j.fct.2012.08.021

Yuan, S.-F., and Alper, H. S. (2019). Metabolic engineering of microbial cell factories for production of nutraceuticals. Microb. Cell Fact. 18:46. doi: 10.1186/s12934-019-1096-y

Conflict of Interest: SH, BD, DK, and IB are named inventors on a European Patent application covering parts of the work described above.

The remaining authors declare that the research was conducted in the absence of any commercial or financial relationships that could be construed as a potential conflict of interest.

Copyright (c) 2019 van der Hoek, Darbani, Zugaj, Prabhala, Biron, Randelovic, Medina, Kell and Borodina. This is an open-access article distributed under the terms of the Creative Commons Attribution License (CC BY). The use, distribution or reproduction in other forums is permitted, provided the original author(s) and the copyright owner(s) are credited and that the original publication in this journal is cited, in accordance with accepted academic practice. No use, distribution or reproduction is permitted which does not comply with these terms. 